\title{
INFLUENCIA DA DEFORMAÇÃO NA OBTENÇÃO DO COEFICIENTE DE ANISOTROPIA NORMAL $r$ E DO COEFICIENTE DE ANISOTROPIA PLANAR $\triangle$ r PARA CHAPAS DA LIGA C22000*
}

Leandro de Almeida ${ }^{1}$ Claudio Geraldo Schön²

\section{Resumo}

Tiras e chapas de cobre e suas ligas possuem excelentes propriedades térmicas, elétricas e mecânicas simultaneamente e por isso podem ser utilizadas na fabricação de inúmeros produtos através de processos de conformação. Determinadas características devem ser analisadas na chapa antes mesmo do processo de manufatura, visando eliminar possíveis falhas durante as etapas de produção. Especificamente para o processo de repuxo profundo, faz-se necessário avaliar a possível formação de orelha e o possível afinamento da espessura da chapa durante a conformação. Sendo assim, o levantamento dos coeficientes de anisotropia planar $\Delta r$ e o de anisotropia normal $r$ são essenciais para se obter bons resultados nos processos de conformação. O presente estudo tem como objetivo avaliar a influência da deformação $(\varepsilon)$ na obtenção dos coeficientes $\Delta r$ e $r$ na liga UNS C22000, utilizada especificamente para a produção de projéteis A norma ASTM E 517 que apresenta a metodologia para se determinar esses coeficientes não especifica claramente qual $\varepsilon$ a ser utilizada. Os resultados alcançados não demonstram diferenças significativas com relação a $\varepsilon$ utilizada nos ensaios, ou seja, os coeficientes obtidos utilizando 0,10, 0,15 e 0,20 de deformação são os mesmos.

Palavras-chave: Conformação; C22000; Anisotropia; Planar; Normal.

INFLUENCE OF STRAIN ON NORMAL ANISOTROPY COEFFICIENT R AND PLANAR ANISOTROPY COEFFICIENT $\triangle R$ FOR C22000 ALLOY SHEET

\section{Abstract}

Strips and plates made of copper and its alloys simultaneously have excellent thermal, electrical and mechanical features, and therefore can be used in the manufacturing of several products through forming processes. Certain features must be analyzed on the plate even before the manufacturing process of the components, in order to eliminate possible failures during the production stages. Specifically for the process of deep drawing, it is necessary to assess the possible ear forming and the possible thinning of the thickness of the sheet after the forming process. Thus, studying the coefficients as the anisotropy planar $\Delta r$, and the anisotropy normal $r$, is essential to obtain good results in the forming process. This study aims at evaluating the influence of the strain in obtaining the $\Delta r$ and $r$ coefficients in the UNS C22000 alloys, used specifically for cartridge production. The ASTM E 517 standard, which shows the methodology for determining these coefficients, does not clearly specify what is the strain to be used. The results obtained do not show significant differences with respect to the strain used in the trials, namely the coefficients can be obtained using other $0,10,0,15$ or 0,20 strain, which resulting values are the same.

Keywords: Forming; C22000; Anisotropy; Planar; Normal.

1 Engenheiro Metalurgista, Engenheiro de Processos, Laminados, PARANAPANEMA S/A., Santo André, São Paulo, Brasil.

2 Físico, Doutor, Livre Docente, Departamento de Engenharia Metalúrgica e de Materiais, Escola Politécnica da Universidade de São Paulo, São Paulo, Brasil. 


\section{INTRODUÇÃO}

O processo de estampagem de cobre e suas ligas é utilizado para a fabricação de inúmeras peças para diversas áreas industriais, como: automobilística, linha branca, instrumentos musicais, conectores elétricos e fabricação de projéteis. Desta forma, faz-se necessário um entendimento aprofundado das propriedades mecânicas indispensáveis para a fabricação de tais itens, assim como ensaios que permitam avaliar tais características e consecutivamente aprovação ou rejeição pela usina durante a manufatura da matéria-prima. A avaliação do coeficiente de anisotropia planar $\Delta r$ nos permite prever uma possível formação de orelha, a qual é originada pela diferença de propriedades mecânicas no plano da chapa, ou seja, existe uma diferença de propriedades nos sentidos $0^{\circ}, 45^{\circ}$ e $90^{\circ}$ de laminação da chapa. Tal característica, quando presente, poderá resultar retrabalho ou rejeição de peças.

O coeficiente de anisotropia normal $r$ também é muito importante, pois avalia um possível afinamento da espessura da chapa após a fabricação do item, podendo comprometer estruturalmente a peça a ser produzida.

A liga de cobre UNS C22000 possui em sua composição química 90\% de cobre e $10 \%$ de zinco. Conhecida comercialmente como Tomback 220, sua principal aplicação é para produção de projéteis, que exigem repuxo profundo. Sendo assim, a formação de orelha e o afinamento da chapa no processo de manufatura desses itens são extremamente indesejáveis, por motivos produtivos e é claro de qualidade do produto final.

A norma ASTM E 517 - "Standard test method for plastic strain ratio $r$ for sheet metal" [1] expõe a metodologia de realização dos ensaios para obtenção do $\Delta r$ e r. Porém, alguns detalhes importantes são deixados a critério dos usuários e não são explicados detalhadamente os possíveis erros ou inconvenientes com os resultados obtidos. A deformação $\varepsilon$ a ser utilizada é um item que a norma não demonstra com tanta clareza e importância. É apresentada apenas uma nota no item 9.2.4, informando que para aço carbono a deformação pode ser de 0,15 a 0,20, e dois exemplos são expostos na nota 2 do item 9.3 .5 com aço e alumínio, demonstrando que pode ocorrer um erro de 0,03 na obtenção do coeficiente de anisotropia normal.

O cálculo dos coeficientes é apresentado claramente pela norma por meio de três equações, sendo a primeira para o cálculo do coeficiente de anisotropia $r$, o segundo para o coeficiente de anisotropia normal e o terceiro para o cálculo do coeficiente de anisotropia planar;

$$
\begin{aligned}
r & =\frac{\ln \left(w_{0} / w_{\mathrm{f}}\right)}{\ln \left(l_{\mathrm{f}} w_{\mathrm{f}} / l_{0} w_{0}\right)} \\
r_{\mathrm{m}} & =\left(r_{0}+2 r_{45}+r_{90}\right) / 4 \\
\Delta r & =\left(r_{0}+r_{90}-2 r_{45}\right) / 2
\end{aligned}
$$

São raras as publicações que expõem a deformação com que os valores de $\Delta r$ e $r$ foram obtidos. A. Melander [2] obteve seus coeficientes para as ligas cobre tough pitch, cobre oxygen-free, latão e cobre-níquel com a deformação variando de 0,15 a 0,20 , expondo apenas essa faixa de deformação, sem maiores detalhes. 
Vial et al. [3] descrevem no artigo "Yield loci of anisotropic sheet metals" uma comparação entre quatro tipos de chapas metálicas: aço desoxidado com alumínio, alumínio 3003-0, cobre 110 e latão 260, com diversas deformações de 0,02 a 0,39, em ensaios de tração unixial, biaxial e de compressão. Porém, não são apresentados detalhes, sendo exposto apenas os resultados e as faixas de deformação utilizadas. Contudo, os autores afirmam que não existe diferença no cálculo de r em função das deformações utilizadas.

J.R. Davis em Tensile Testing [4] expõe que alguns trabalhos sugerem que os ensaios sejam feitos em uma deformação mínima de 0,15 , não especificando quais trabalhos nem tão pouco em quais materiais devem ser empregados essa deformação. $O$ autor deixa claro que o valor de $r$ geralmente não se altera muito durante o ensaio de tração uniaxial, pois essa deformação de 0,15 é suficientemente grande para ser efetuada a medição com uma precisão razoável.

Frącz et al. [5] publicaram recentemente em 2014 um estudo sobre a comparação da estampabilidade entre uma super-liga a base de níquel AMS 5596, utilizada em aplicações aeroespaciais, e o aço EDDQ utilizado na indústria automobilista. Graficamente, a influência da deformação na obtenção do coeficiente de anisotropia r é bastante notória, sendo o comportamento das ligas bastante diferente entre si.
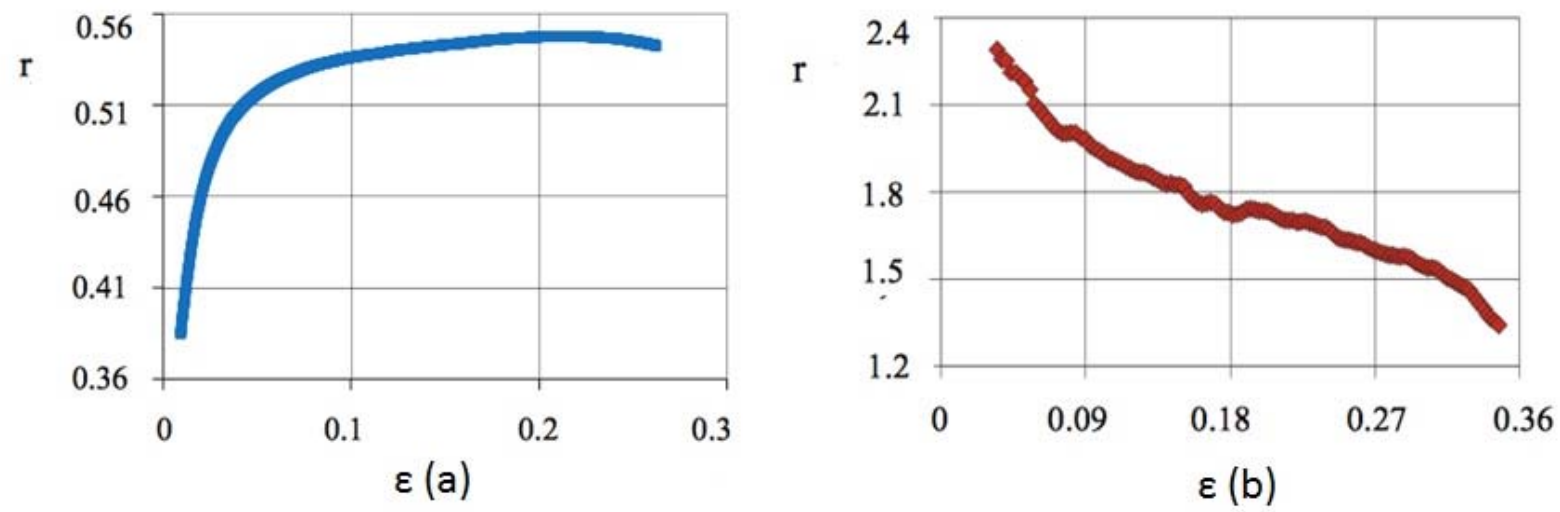

Figura 01 - Gráficos demonstrando o coeficiente de anisotropia normal $x \varepsilon$ da super-liga de níquel ASM 5596 (a) e do aço EDDQ (b) (adaptado de [5]).

\section{MATERIAIS E MÉTODOS}

As amostras disponibilizadas para a realização dos ensaios foram doadas pela PARANAPANEMA S/A com espessura de $1,90 \mathrm{~mm}$ na têmpera mole, sendo a composição química atestada em certificado de qualidade pelo fabricante como liga UNS C22000.

A preparação da amostra para metalografia foi efetuada com um corte no sentido de laminação e dois transversais, gerando uma amostra de 20X10X1,90 mm. A amostra foi embutida de topo em baquelite com diâmetro de $25 \mathrm{~mm}$ e altura de $18 \mathrm{~mm}$. Após o embutimento, realizou-se o lixamento da amostra utilizando lixa de carbeto de silício de granulometria de \#120, \#320, \#400 e \#500. O polimento foi realizado com pano em suspensão de diamante de 6 e $1 \mu \mathrm{m}$. O ataque químico foi realizado com uma solução de Trióxido de Cromo por 5 segundos e a remoção da solução efetuada com álcool e água corrente, seguido da secagem com soprador de ar quente.

O corpo de prova metalográfico foi analisado no microscópio óptico Olympus modelo BX-60M, com aumento de 75 e 100X e a medição do tamanho de grão foi efetuado conforme o método comparativo disponível na norma ASTM E112 [6]. 
O ensaio de obtenção das propriedades mecânica de limite de resistência à tração, limite de escoamento e alongamento foram realizados na máquina de ensaios universal do tipo Kratos modelo K20000MP para $2000 \mathrm{kgf}$, com o extensômetro da Kratos modelo E-10 com abertura inicial de $25 \mathrm{~mm}$. A velocidade de deformação usada foi de $6 \mathrm{~mm} / \mathrm{min}$ e a pré-carga de $0,50 \%$, os dados obtidos nos ensaios foram analisados com o auxilio do software Excel.

Três chapas de 400X400X1,90 mm foram recortadas em uma guilhotina do centro da chapa padrão de $650 \times 650 \times 1,90 \mathrm{~mm}$ fornecida pela PARANAPANEMA S/A. Os cortes foram efetuados visando a centralização da chapa na máquina de eletroerosão (Hitech nc machine tool co. Itd - Dk7632), responsável pelo corte dos corpos de prova nos três sentidos $0^{\circ}, 45^{\circ}$ e $90^{\circ}$ com dimensional demonstrado na norma ASTM E 517 [1], conforme a Figura 2.

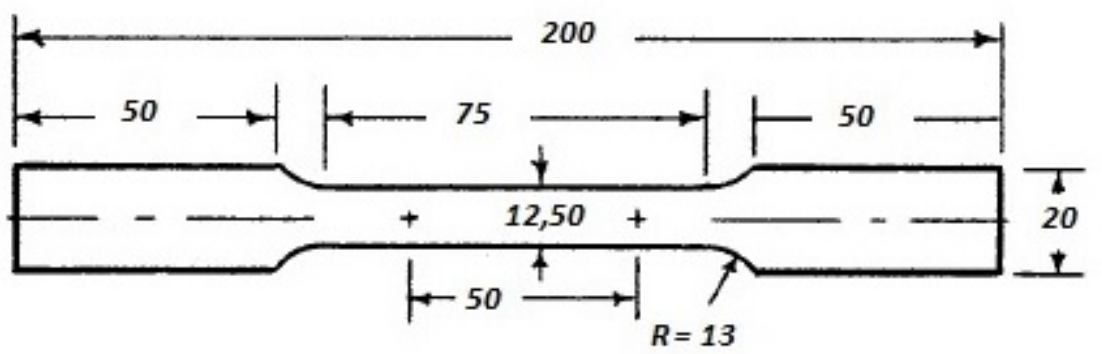

Figura 02 - Corpo de prova tipo gravata utilizado nos ensaios, conforme norma ASTM E 517, dimensionais em mm (Adaptado de [1]).

Foram confeccionados 9 corpos de prova de cada ângulo, totalizando 27 corpos de prova, objetivando avaliar 3 corpos de prova por ângulo e deformação. As deformações foram confirmadas após os ensaios por meio da medição no próprio corpo de prova do afastamento entre riscos previamente espaçados a $20 \mathrm{~mm}$.

Com um paquímetro Mitutoyo modelo 500-197-30B digital com resolução de 0,01 $\mathrm{mm}$, realizou-se as medições de largura e comprimento nas regiões demarcadas nos corpos de prova antes e após os ensaios. A medição das espessuras foi efetuada com um micrômetro também da marca Mitutoyo modelo 395-251 digital com resolução de $0,001 \mathrm{~mm}$, antes e após os ensaios.

Os ensaios realizados são semelhantes aos de tração uniaxial, porém, os corpos de prova não sofrem o rompimento e o ensaio é interrompido de acordo com a deformação objetivada.

\section{RESULTADOS E DISCUSSÃO}

A composição química da chapa fornecida pela PARANAPANEMA S/A. apresentou uma somatória de impureza extremamente baixa, 0,0170\%, conforme a Tabela 1.

Tabela 1. Composição química da liga UNS C22000

\begin{tabular}{lccccc}
\hline LIGA & \% Cu & \% Zn & \% Fe & \% Sn & \% Cr \\
\hline C22000 & 89,980 & 10,003 & 0,0051 & 0,0043 & 0,0076 \\
\hline
\end{tabular}

Foram efetuados ensaios de tamanho de grão através do método comparativo disponibilizado pela ASTM E112 [6], sendo detectado um tamanho de grão médio de $0,040 \mathrm{~mm}$. A Figura 03 apresenta uma imagem utilizada na avaliação de tamanho de grão. 


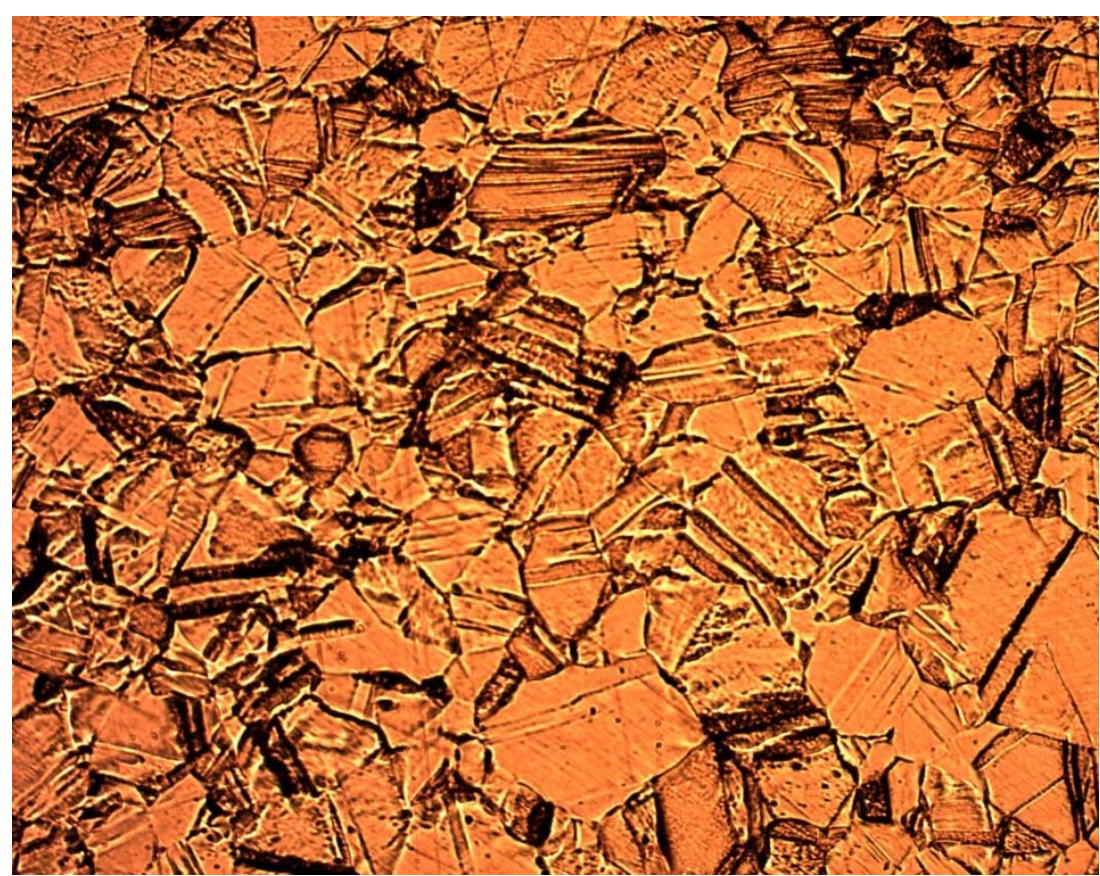

Figura 03 - Metalografia da chapa cedida pela PARANAPANEMA S/A., apresentando fase a e tamanho de grão médio de $0,040 \mathrm{~mm}$.

Os ensaios mecânicos foram realizados conforme a norma ASTM E8 [7], para a obtenção do limite de resistência à tração, limite de escoamento com $0,02 \%$ e alongamento em 50,80 mm, no sentido de laminação $0^{\circ}$. Os resultados são apresentados na Tabela 2.

Tabela 2. Resultado das propriedades mecânicas obtidas.

\begin{tabular}{cccc}
\hline AMOSTRA & $\begin{array}{c}\text { Média } \\
\text { LRT } \\
(\mathbf{M P a})\end{array}$ & $\begin{array}{c}\text { Média L.esco. } \\
(\mathbf{0 , 0 2 \% )}(\mathbf{M P a})\end{array}$ & $\begin{array}{c}\text { Média Along. } \\
\text { (em 2) } \\
(\%)\end{array}$ \\
\hline C22000 & 231 & 60 & 39,8 \\
\hline
\end{tabular}

Os parâmetros n, coeficiente de encruamento, e K, coeficiente de resistência, ambos da equação de Zener-Hollomon, também foram avaliados em três amostras, haja vista que são importantes para a avaliação da estampabilidade das chapas. Os resultados são apresentados na Tabela 3.

Tabela 3. Resultado dos parâmetros $n$ e K da equação de Zener-Hollomon.

\begin{tabular}{ccc}
\hline AMOSTRA & Média $\mathbf{n}$ & Média K (MPa) \\
\hline C22000 & 0,5437 & 520 \\
\hline
\end{tabular}

Os resultados médios de três amostras dos coeficientes de anisotropia planar $\Delta \mathrm{r}$ e dos coeficientes de anisotropia normal $r$ para cada deformação $\varepsilon$, são demonstrados na Tabela 4.

Tabela 4. Resultado (médias) dos coeficientes de anisotropia planar e normal.

\begin{tabular}{ccc}
\hline $\boldsymbol{\varepsilon}$ & $\Delta \mathbf{r}$ & $\mathbf{r}$ \\
\hline 0,1 & $-0,37$ & 1,00 \\
\hline 0,15 & $-0,40$ & 1,04 \\
\hline 0,2 & $-0,40$ & 1,04 \\
\hline
\end{tabular}


Os valores de $r$ calculados para as deformações $\varepsilon=0,10, \varepsilon=0,15$ e $\varepsilon=0,20$ apresentaram uma variação de 0,04 entre os valores obtidos. Analisando os resultados dos 9 coeficientes $r$ calculados, obtém-se um desvio padrão de 0,04 , ou seja, os valores dos coeficientes de anisotropia normal não apresentaram grandes variações, pois a variação total entre os valores obtidos é a mesma do desvio padrão obtido.

Os coeficientes de anisotropia planar $\Delta r$ não apresentaram grandes variações, pois da mesma forma que o coeficiente de anisotropia normal $r$, apresentaram uma variação de 0,03 com um desvio padrão obtido entre os 9 valores calculados de 0,04, demonstrando também que a deformação não influencia o cálculo dos coeficientes para a liga de cobre C22000 estudada.

J.R. Davis [4] menciona em Tensile Testing que medidas de $r$ em alongamentos e consecutivamente em deformações menores que 0,15 apresentam uma precisão menor na obtenção dos resultados. Durante a realização dos ensaios, pode-se observar que os materiais mais deformados, $\varepsilon=0,15$ e $\varepsilon=0,20$, apresentaram uma variação menor nas medições de largura e espessura, corroborando com as resultados de J.R. Davis afirma [4].

\section{CONCLUSÃO}

Diante do exposto, evidencia-se que os objetivos almejados foram alcançados, pois foi possível demonstrar que para a liga de cobre UNS C22000, a deformação utilizada no cálculo dos coeficientes de anisotropia planar $\Delta r$ e normal $r$ não apresentou influência significativa na obtenção dos resultados, haja vista que as variações foram iguais ou menores que o próprio desvio padrão obtido nas amostras. Porém, com $\varepsilon=0,10$ notou-se uma variação maior na obtenção dos resultados devido ao próprio método de medição.

\section{Agradecimentos}

A empresa PARANAPANEMA S/A., pela colaboração, disponibilidade de amostras fornecidas e utilização dos equipamentos para ensaios.

\section{REFERÊNCIAS}

1 States. 2010.

2 MELANDER, A. A new model of the forming limit diagram applied to experiments on four copper-base alloys, Materials science and engineering, 58, p. $63-88,1983$.

3 VIAL, C.; HOSFORD, F., W.; CADDELL, R., M. Yield loci of anisotropic sheet metals, Int. J. Mech. Sci., Vol. 25, No. 12, p. $899-915,1983$.

4 DAVIS, J. R. ASM Speciality Handbook - Tensile testing, Second Edition, USA, ASM International, 2004.

5 FRACZ, W.; STACHOWICZ, F.; TRZEPIECINSKI, T.; PIEJA, T. Formability of the AMS 5596 sheet in comparison with EDDQ steel sheet, Journal of Mechanics Engineering and Automation, Vol. 4, p. 72 - 77, 2014.

States. 2013

E 112: Standard Test Method for Determining Average Grain Size. United

7
E 8: Standard Test Method for Tension Testing of Metallic Materials. United 